\title{
Evaluation of French Motorway Network in Relation to Slime Mould Transport Networks
}

\author{
Andrew Adamatzky ${ }^{1}$, Olivier Allard ${ }^{2}$, Jeff Jones ${ }^{1}$ and Rachel \\ Armstrong $^{3}$ \\ ${ }^{1}$ Unconventional Computing Centre, UWE, Bristol, UK \\ ${ }^{2}$ Biomimesis, Courbevoie, France \\ ${ }^{3}$ School of Architecture, Planning and Landscape, Newcastle \\ University, UK
}

April 21, 2017

\begin{abstract}
France has developed a high quality motorway system that has been rapidly rationalized and matured in the late $20^{\text {th }}$ century yet has been founded on ancient, Roman infrastructures. The development of the motorway system is thus an iterative method associated with hierarchical 'top-down' processes taking into consideration factors such as population density, network demand, location of natural resources, civil engineering challenges and population growth. At the opposite extreme to this approach is the development of transport networks within simple biological systems which are typically decentralised, dynamic and emerge from simple, local and 'bottom-up' interactions. We examine the notion, and to what extent, that the structure of a complex motorway network could be predicted by the transport network of the single-celled slime mould Physarum polycephalum. This comparison is explored through its ability to 'deduce' the French motorway network in a series of analogue and digital experiments. We compare Physarum network and motorway network topology in relation to proximity graphs and assess the trade-off between connectivity and minimal network length with a bottom-up model of a virtual plasmodium. We demonstrate that despite the apparent complexity of the challenge, Physarum can successfully apply its embodied intelligence to rationalise the motorway topology. We also demonstrate that such calculations prove challenging in the face of significant obstacles such as, mountainous terrain and may account for the missing route between Nice, Grenoble, Avignon and Lyon. Finally, we discuss the topological findings with respect to circle and spoke city planning infrastructures and certain species of web-building spiders.
\end{abstract}

\section{Introduction: The French Motorway Network}

The French motorway network is the fourth largest in the world comprising $11,882 \mathrm{~km}$ length of motorways, including $9048 \mathrm{~km}$ of toll highway (see data 
from ASFA (2014)). It takes almost 25 percent of the national traffic while accounting for only one percent of French roads in linear distance Gandil (2005). Underpinning the success of these motorways is that some regions like Corsica and a large part of Brittany do not have highways but expressways. Only two French territories are not served by any highway namely, Ardèche and HautesAlpes. This is in part due to the mountainous terrain which provides a significant engineering obstacle and there is therefore no direct highway connecting the two major cities Nice and Lyon.

France's motorway system has ancient origins - the first Roman roads of northern Gaul were established by Emperor Augustus (63 B.C. to 14 A.D.), who entrusted this task to his son-in-law Agrippa (62 B.C. to 12 B.C.). He started then the creation of a vast hub-and-spoke network centred on Lyon, the new capital of Gaul, thus joining the confines of the Empire to the north, west and south. Only twenty-five years after the end of the Gallic Wars, Rome was connected to the English Channel by a route for the military and commercial operation of its new conquests. Yet, the initial plan was not fully completed until the middle of the $1^{\text {st }}$ century A.D. During the four centuries that followed, new important towns were connected to this infrastructure, or re-routed to bypass unsafe or poorly maintained roads.

However, even the Roman roads have more ancient origins that rationalised a dense, heterogeneous and well maintained Gallic network of roads that were shaped by independent trading routes between hundreds of local territories. This refined network enabled Roman armies to move more efficiently and communicate more effectively with its imperial power centres. In peacetime, the network promoted the development of trade and civilian communications between different parts of the Roman Empire. The roads were particularly useful for grain supply of the large urban centres, which were swelling significantly during antiquity. The disappearance of the imperial administration in late antiquity led, among other things, to termination of road maintenance. After the ravages of the Hundred Years' War (1337-1453), the creation of a powerful monarchy allowed the reconstruction of the country with Paris as the centre and the rehabilitation of roads and land routes. The road network became a priority of the state during the $18^{\text {th }}$ century with the "Ponts et Chaussées" ("Bridges and Roads") service being created in 1716 to develop the road network with the support of the Ministers of War. Up until the French Revolution around 30,000 kilometres of roads were created under the Royal Chore. This made incumbent French regimes responsible for their maintenance - initially royal, then imperial under Napoleon, and finally the duty of national government in the $20^{\text {th }}$ century that has been underpinned by a stable form of private/public financing Gandil (2005).

French motorways are therefore both imprints of history and the logical consequence of network optimization. Generally, they have been built in parallel with many national roads and in some cases they allow for more direct routes to major cities - including the displacement of the centre of gravity from Lyon to Paris. A further complexification of the French motorway network is conferred by its connection to sparsely populated cities, which is due to geographic, economic or historical reasons and makes the reading of the French network more 


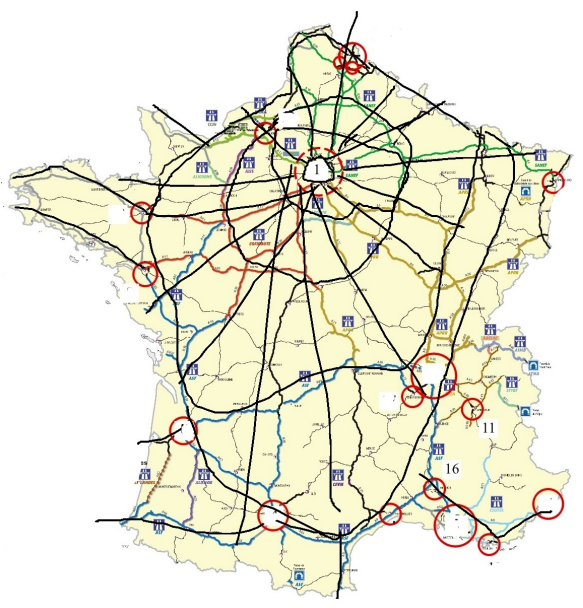

Figure 1: Motorway network in mainland France is reminiscent of spider-web structure.

complex.

The modern rationalisation of French motorways began in the late $20^{\text {th }}$ century. Between 1960 and 1970, the network grows from Paris to Lille, in the north, Paris to Marseille-Toulon, in the south and bypasses Dijon, Lyon and Avignon. Paris-Lyon-Marseille is a major structural axis in France. The motorway linking Paris to Rouen, to the west, is also built during the 60s. During the $70 \mathrm{~s}$ and the $80 \mathrm{~s}$, the network extends radially from Paris to cities of Strasbourg in the east, Nantes and Bordeaux in the southwest, Le Havre in the west, and Clermont-Ferrand in the centre of France. During this time, the Paris-Marseille motorway is extended westward to Toulouse via Montpelier. Between 1990 and 2000, these axes leaving Paris are extended from the cities in the centre of France to the major southern cities. The concentric orbits around Paris that form its current, striking 'spider-web' configuration (Fig. 1) begin in 2000 and are ongoing, where radial axes join the major French cities without passing through Paris, such as the Lyon-Bordeaux and Nantes-Bordeaux motorways.

\section{Slime Mould Transport Networks}

The emerging influence of centralised large scale planning, coordination and control of the motorway network is in contrast to the construction of transport networks employed by a wide range of simple organisms Bebber, Hynes, Darrah, Boddy, and Fricker (2007). Like human constructs, these networks are used to transport material (for example, nutrients, metabolites) about the organism Fricker, Bebber, and Boddy (2008) or population Garnier, Guérécheau, Combe, Fourcassié, and Theraulaz (2009). In this paper we concentrate on transport networks formed by an extremely simple single-celled organism.

Over a range of theoretical and experimental works the true slime mould Physarum polycephalum has demonstrated that it can be manipulated and used in an 'unconventional' A. Adamatzky, Bull, and Costello (2007) computational context to solve mazes Nakagaki, Yamada, and Toth (2000), 'remember' environ- 
mental details Reid, Latty, Dussutour, and Beekman (2012); Saigusa, Tero, Nakagaki, and Kuramoto (2008) and also find the shortest routes between spatially separated points A. Adamatzky (2010, 2012); Shirakawa, Adamatzky, Gunji, and Miyake (2009).

Physarum polycephalum is a macroscopic protist belonging to the phylum mycetozoa. It is visible to the naked eye and typically grows to over $10 \mathrm{~cm}^{2}$ The organism consists of a giant single cell containing myriad nuclei, during its vegetative stage, which is able to move via contractile protein-induced oscillations in its gel-sol core Durham and Ridgway (1976). Under hydrostatic pressure from these contractions, the active growth front of the organism streams forward, attracted by nearby nutrient gradients. Behind the densely ramified growth front, the structure of the plasmodium adapts and coarsens, forming a protoplasmic tube connecting the growth front and previously engulfed nutrients. These tubes are used to distribute nutrients throughout the entire organism. In this state it has been considered capable of demonstrating 'intelligent behaviour' although it lacks any typical centrally organised structure to coordinate these tasks such as, a brain, or formally organised nervous system. Such an information processing system may be regarded as a form of embodied intelligence A. Adamatzky (2012) that is able to resolve internal and external cues through morphology.

It's ability to compute transport network topologies resides in plasmodium's ability to construct and adapt its self-made and self-organised transport network when presented with a choice of food sources (typically oat flakes in experimental conditions). As it forms a set of communicating tubes to distribute nutrients and links its initial and final destination. Consequently, an optimized network of tubes is formed when several food sources are presented. In this manner Physarum exhibits a basic form of decision making when establishing the distribution of these tubes. Typically the plasmodium body will engulf smaller obstacles but spread around larger ones and effectively perform a complex set of manoeuvres that calculate the most efficient network topology for the organism and approximates man-made travel routes A. Adamatzky and Jones (2010); A. I. Adamatzky (2014). Corresponding city locations can be represented on a nutrient medium using food sources, which are typically provided by natural bacterial colonies that grow on oat flakes.

While such maps have been produced for some countries A. Adamatzky (2012) and individual cities Tero et al. (2010), no such biologically inspired adaptive network modelling of the French motorway exists. French motorways offer a particularly intriguing challenge for the network rationalization powers of Physarum since they have a complex origin established during Roman times that has been rationalised over the late $20^{\text {th }}$ century.

In this paper we will examine the established ability A. Adamatzky (2012); A. Adamatzky et al. (2013) of the plasmodium to map transport routes between hubs of activity that represent cities. In these instances, iterative exchanges between the Physarum body and complex factors in the environment provide a model through which it is possible to find the shortest route between two points to generate network topologies with comparable efficiency, fault tolerance, and cost to those of real-world infrastructure networks Tero et al. (2010).

Specifically, we will compare the structure of slime mould Physarum networks connecting analogues of major urban areas of mainland France with the French motorway network connecting the same areas. We will compare the similarity and differences of Physarum networks and motorway networks. The 


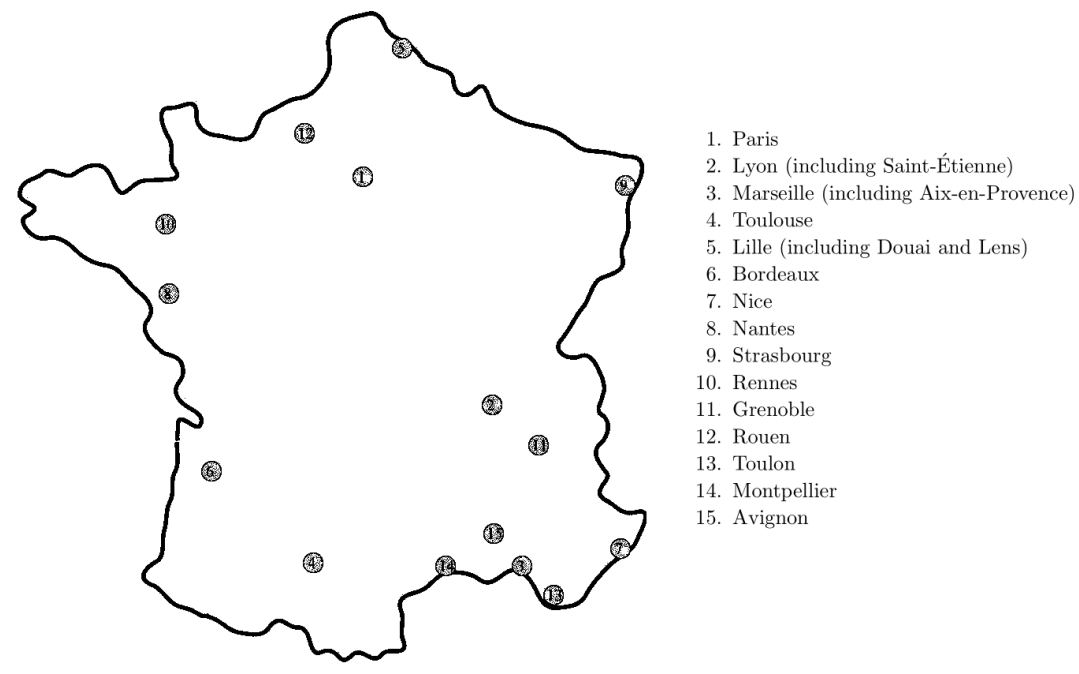

Figure 2: Outline map of France with major urban areas $\mathbf{U}$ shown by encircled numbers.

structure of the paper progresses as follows: Section 3 describes the experimental methods to construct Physarum networks. Section 4 describes the process of statistically quantifying the Physarum network structure from the experimental results. The Physarum networks are statistically compared to the motorway network in section 5 and both Physarum and motorway networks are compared to proximity graph structures in section 6. A modelling approach using the bottom-up network adaptation processes seen in slime mould is described in section 7. Finally, a discussion of the findings is given in section 8

\section{$3 \quad$ Experimental methods}

Plasmodium of $P$. polycephalum is cultivated in plastic container, on paper kitchen towels moistened with still water, and fed with oat flakes. For experiments we use $220 \times 220 \mathrm{~mm}$ polystyrene square Petri dishes and $2 \%$ agar gel (Select agar, by Sigma Aldrich) as a substrate. Agar plates, about 2-3 mm in depth, are cut in a shape of continental (mainland) France.

We selected the 15 most populated major urban areas of France (see configuration in Fig. 2, which roughly corresponds to distribution of population densities). The size of each oat flake was approximately equal (5mm) and did not directly correspond to the relative size of the selected urban areas.

To represent the set of major urban areas, denoted by $\mathbf{U}$, we place oat flakes in the positions of agar plate corresponding to the areas. At the beginning of each experiment an oat flake colonised by plasmodium is placed in the Paris area. We undertook 12 experiments. The Petri dishes with plasmodium are kept in darkness, at temperature $22-25^{\circ} \mathrm{C}$, except for observation and image recording. Periodically, the dishes are scanned with an Epson Perfection 4490 scanner and configurations of protoplasmic networks analysed. A typical image of an experimental Petri dish is shown in Fig. 3. 


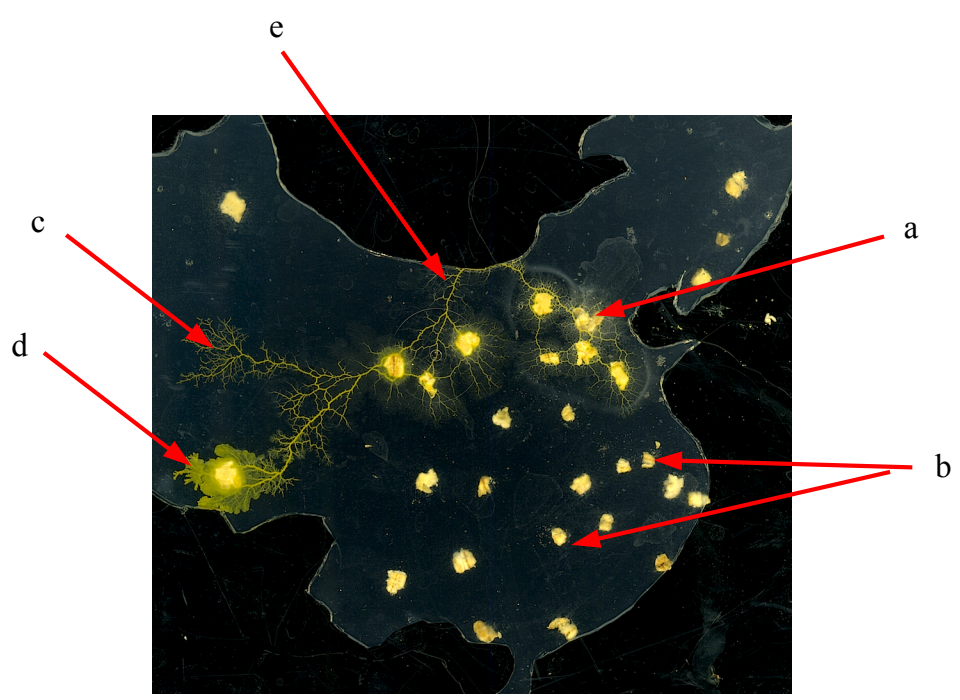

Figure 3: A typical image of slime mould $P$. polycephalum growing on a nonnutrient substrate and connecting oat flakes, which represent major urban areas $\mathbf{U}$ by a network of protoplasmic tubes. a: site of inoculation, b: virgin oat flakes, c: active zone, propagating part of plasmodium in a search for nutrients, d: oat flake occupied by plasmodium's active zone, e: protoplasmic tube.

\section{Results of laboratory experiments}

\subsection{Formation of protoplasmic networks}

In a few hours after inoculation in Paris, the plasmodium recovers from initial shock, starts exploring its substrate, detects gradients of chemo-attractants emitted by virgin oat flakes placed in major urban areas $\mathbf{U}$ and starts propagating along gradients of chemo-attraction. A typical scenario of plasmodium development is shown in Fig. 4 .

On the first day of such a typical scenario the slime mould propagates from its inoculation site in Paris to Rouen and Lille (Fig. 4a). The second day shows growth from Lille to Strasbourg and Lyon, and then from Strasbourg to Grenoble and from Lyon to Grenoble. These are followed by further propagation towards Toulon and Nice (Fig. 4 c). After colonising Nice and Toulon- on the third day of the experiment - the slime mould connects cluster of urban areas Grenoble, Avignon, Marseille, Toulon, Nice and then moves from Avignon to Toulouse, from Montpellier to Toulouse, from Toulouse to Bordeaux, from Bordeaux to Nantes and from Nantes to Rennes (Fig. 4.). On the fourth day of the experiment, the slime mould returns to its original site of inoculation by propagating from Rennes to Rouen to Paris (Fig. $4 \mathrm{~d}$ ).

\subsection{Physarum graphs}

To generalise our experimental results we constructed a Physarum graph with weighted-edges. A Physarum graph is a tuple $\mathbf{P}=\langle\mathbf{U}, \mathbf{E}, w\rangle$, where $\mathbf{U}$ is a set of urban areas, $\mathbf{E}$ is a set edges, and $w: \mathbf{E} \rightarrow[0,1]$ associates each edge of $\mathbf{E}$ with a 


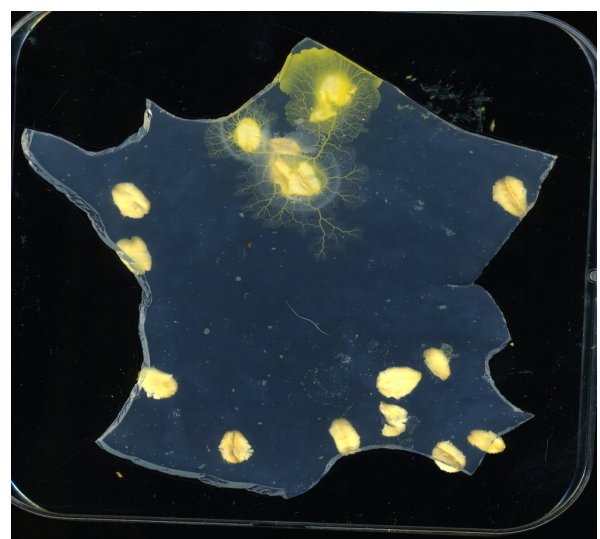

(a) $24 \mathrm{~h}$

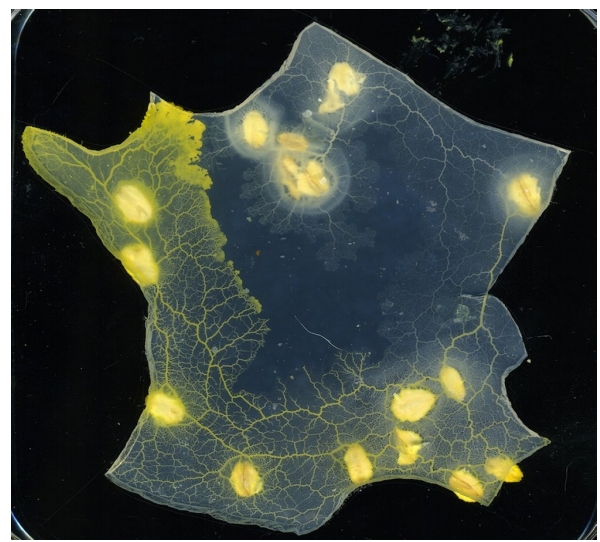

(c) $72 \mathrm{~h}$

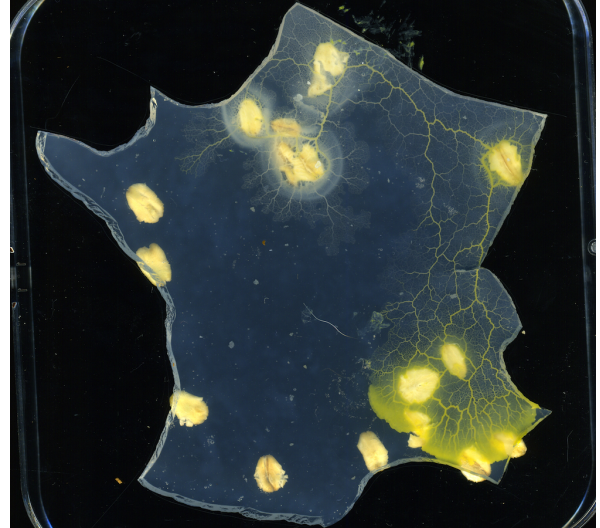

(b) $48 \mathrm{~h}$

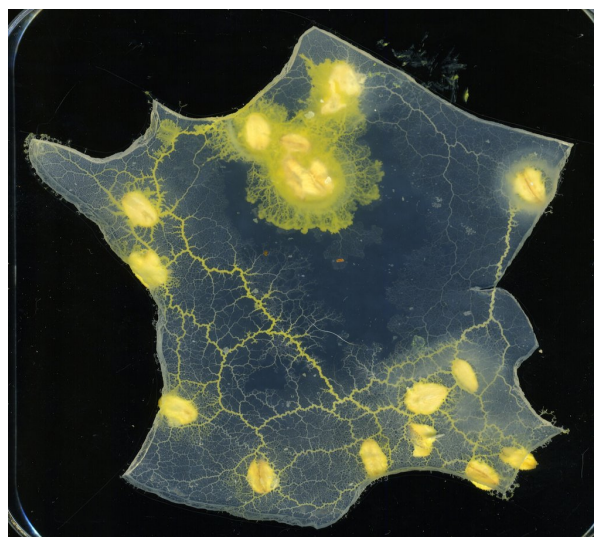

(d) $96 \mathrm{~h}$

Figure 4: Experimental laboratory example of slime mould propagation guided by shape of France and configuration of urban areas U. Petri dishes with slime mould were scanned every $24 \mathrm{~h}$. 


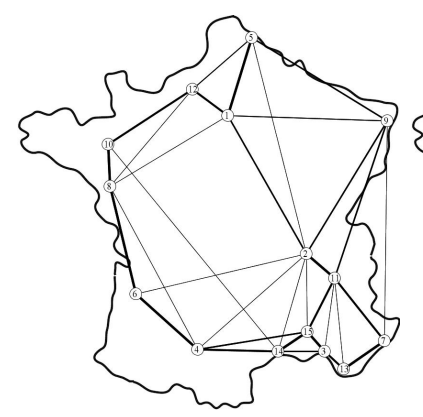

(a) $\theta=\frac{0}{12}$

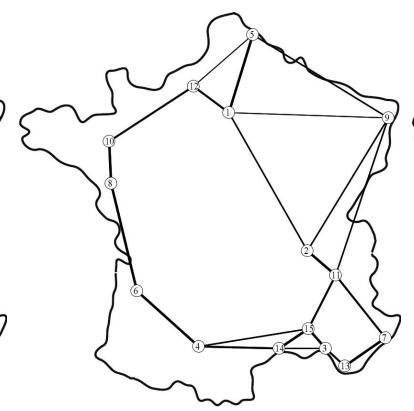

(b) $\theta=\frac{3}{12}$

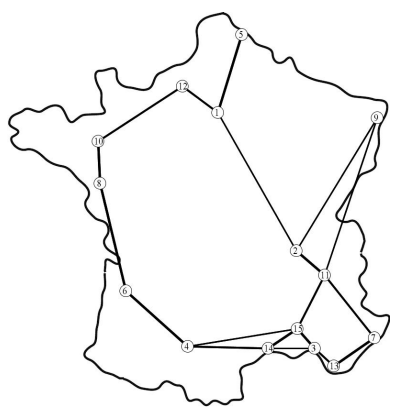

(c) $\theta=\frac{6}{12}$

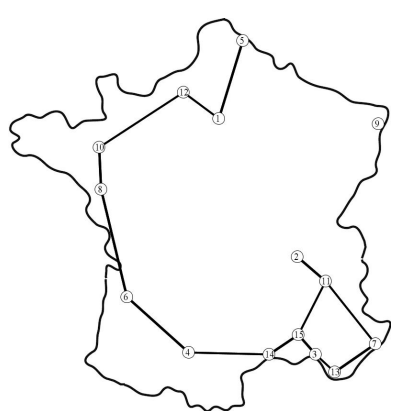

(d) $\theta=\frac{7}{12}$

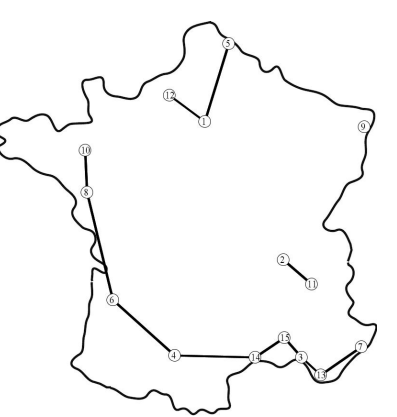

(e) $\theta=\frac{8}{12}$

Figure 5: Generalized Physarum graphs $\mathbf{P}(\theta)$ for selected values of $\theta$. 
probability (or weights). For every two regions $a$ and $b$ from $\mathbf{U}$ there is an edge connecting $a$ and $b$ if a plasmodium's protoplasmic link is recorded at least in one of $k$ experiments, and the edge $(a, b)$ has a probability calculated as a ratio of experiments where protoplasmic link $(a, b)$ occurred in the total number of experiments $k=12$. For example, if we observed a protoplasmic tube connecting areas $a$ and $b$ in 7 experiments, the weight of edge $(a, b)$ will be $w(a, b)=\frac{7}{12}$. We do not take into account the exact configuration of the protoplasmic tubes but merely their existence. Furthermore we will be dealing with threshold Physarum graphs $\mathbf{P}(\theta)=\langle\mathbf{U}, T(\mathbf{E}), w, \theta\rangle$. The threshold Physarum graph is obtained from Physarum graph by the transformation: $T(\mathbf{E})=\{e \in \mathbf{E}: w(e) \geq \theta\}$. That is, all edges with weights less than $\theta$ are removed. Examples of threshold Physarum graphs for various values of $\theta$ are shown in Fig. 5.

In analysing the thresholded results we can see that the Physarum graph undergoes the following critical transformations with increase of $\theta$ :

- $\theta=3$ : The graph becomes planar.

- $\theta=6$ : Leaves (nodes of degree one) appear, e.g. Lille becomes a leaf.

- $\theta=7$ : Isolated nodes appear, e.g. Strasbourg loses connections to the rest of $\mathbf{U}$; Lille and Lyon become leaves.

- $\theta=8$ : The graph splits into four disconnected components. The components are chains Lille- Paris- Rouen, Lyon- Grenoble, Nice- ToulonMarseille, Avignon- Montpellier- Toulouse- Bordeaux- Nantes- Rennes.

The strongest components of the graph are links Nantes- Rennes, ToulouseBordeaux, Marseille--Avignon, Nice- Toulon. They remain in the graph up to $\theta=11$.

\section{Comparative analysis with Mainland France Motorway Network}

To quantify any similarity between Physarum networks and the Motorway network the generalised Physarum graph was compared with the motorway map $\mathbf{H}$. The motorway graph is derived as follows. Let $\mathbf{U}$ be a set of urban regions/cities; for any two regions $a$ and $b$ from $\mathbf{U}$, the nodes $a$ and $b$ are connected by an edge $(a b)$ if there is a highway starting in vicinity of $a$, passing in vicinity of $b$, and not passing in vicinity of any other urban area $c \in \mathbf{U}$. In the case of branching - that is, a highway starts in $a$, goes in the direction of $b$ and $c$, and at some point branches towards $b$ and $c$ - we then add two separate edges $(a b)$ and $(a c)$ to the graph $\mathbf{H}$. The highway graph is planar (Fig. 6).

Intersection of Physarum graph for several values of $\theta$ and the motorway graph is shown in Fig. 7. As we can see, strong components of the Physarum graph are the subgraphs of the France motorway graph.

We can define a degree of approximation of $\mathbf{H}$ by $\mathbf{P}\left(\frac{\theta}{12}\right.$ as a percentage of edges of $\mathbf{H}$ represented by edges of $\mathbf{P}\left(\frac{\theta}{12}\right)$. Then the exact value $\frac{\theta}{12} \cdot 100 \%$ characterises the accuracy of approximation. The plot of the degree of approximation $\delta$ versus accuracy of approximation $\alpha$ is shown in Fig. 8. It fits well a linear approximation $\delta=79-0.62 \cdot \alpha$, with coefficient of determination $R^{2}=0.96$. One of 


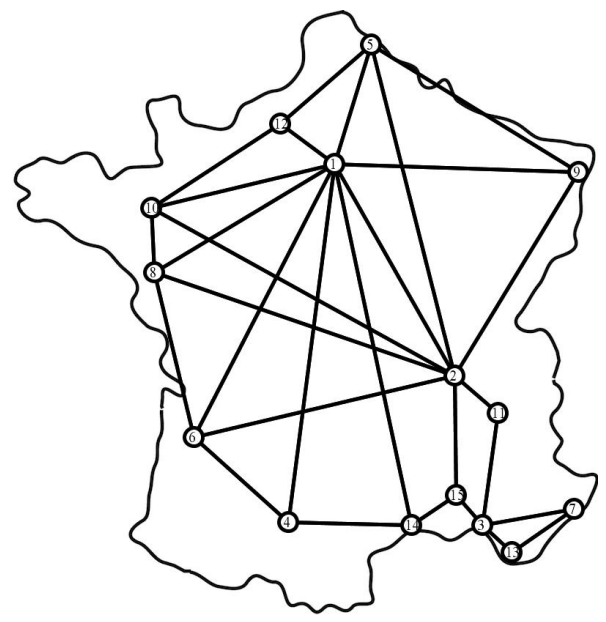

Figure 6: Motorway graph $\mathbf{H}$ of France.

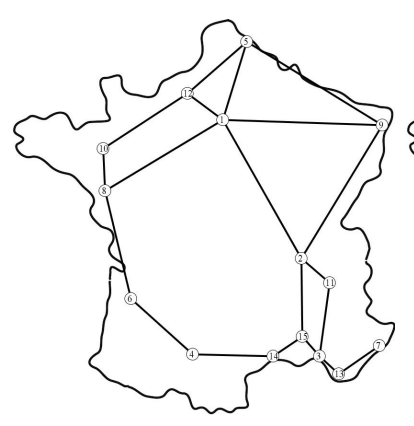

(a) $\mathbf{H} \cap \mathbf{P}\left(\frac{1}{12}\right)$

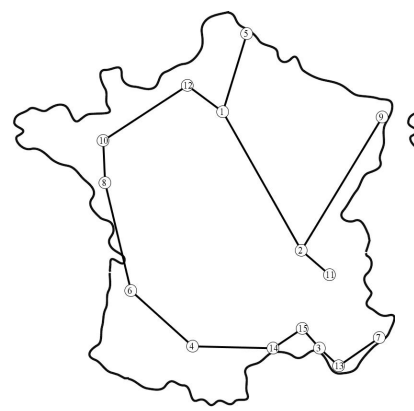

(d) $\mathbf{H} \cap \mathbf{P}\left(\frac{6}{12}\right)$

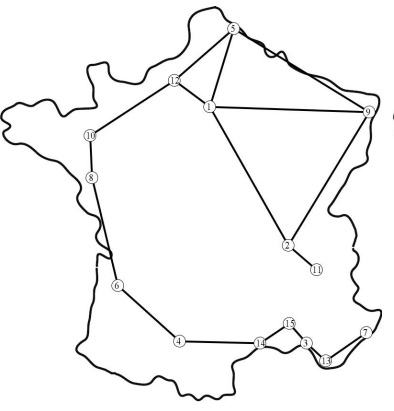

(b) $\mathbf{H} \cap \mathbf{P}\left(\frac{3}{12}\right)$

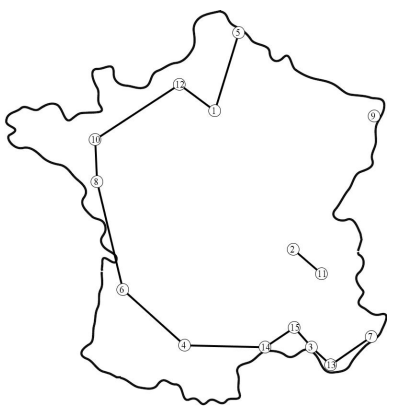

(e) $\mathbf{H} \cap \mathbf{P}\left(\frac{7}{12}\right)$

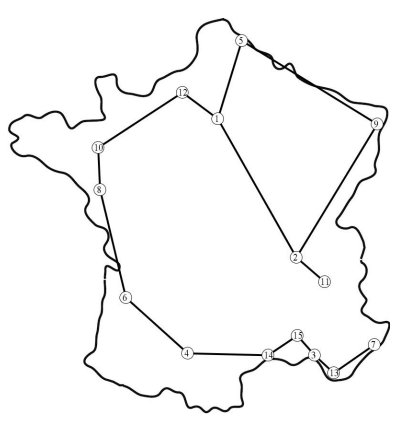

(c) $\mathbf{H} \cap \mathbf{P}\left(\frac{5}{12}\right)$

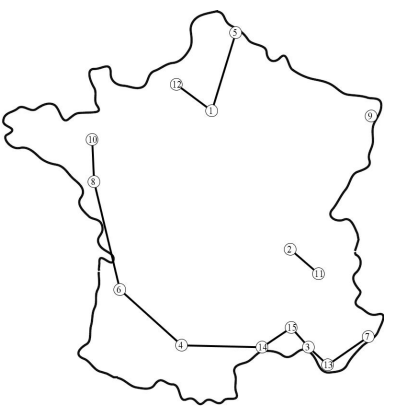

(f) $\mathbf{H} \cap \mathbf{P}\left(\frac{8}{12}\right)$

Figure 7: Intersection of motorway graph $\mathbf{H}$ and Physarum graphs $\mathbf{P}(\theta)$ for (a) $\theta=\frac{1}{12}$, (b) $\theta=\frac{3}{12}$, (c) $\theta=\frac{5}{12}$, (d) $\theta=\frac{6}{12}$, (e) $\theta=\frac{7}{12}$, (f) $\theta=\frac{8}{12}$ 


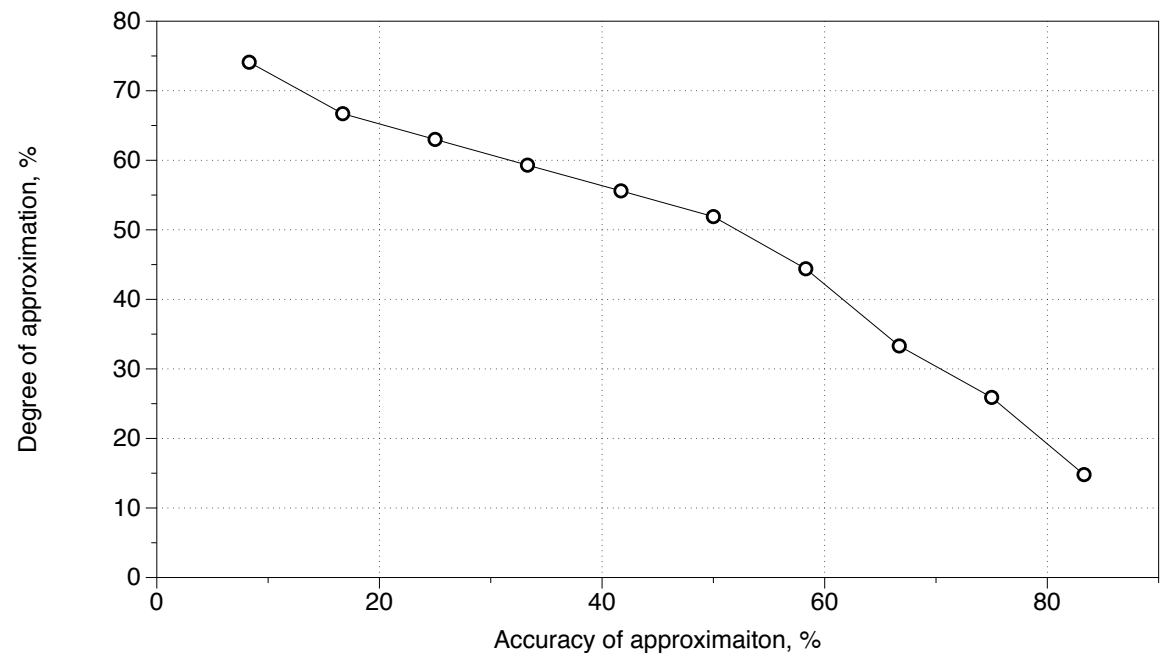

Figure 8: Plot of the accuracy of approximation of France motorway edges by edges of Physarum graphs versus the degree of approximation.

the outcome of the approximation is that Physarum successfully approximates over half of motorway links in over half of the laboratory experiments.

\section{Slime Mould Networks and Motorway Net- works vs Proximity Graphs}

A planar graph consists of nodes which are points of the Euclidean plane and edges which are straight segments connecting the points. A planar proximity graph is a planar graph where two points are connected by an edge if they are close in some sense. A pair of points is assigned a certain neighbourhood, and points of the pair are connected by an edge if their neighbourhood is empty. Here we consider the most common proximity graph as follows.

- GG: Points $a$ and $b$ are connected by an edge in the Gabriel Graph GG if disc with diameter $\operatorname{dist}(a, b)$ centred in middle of the segment $a b$ is empty Gabriel and Sokal (1969); Matula and Sokal (1980) (Fig. 9a).

- RNG: Points $a$ and $b$ are connected by an edge in the Relative Neighbourhood Graph RNG if no other point $c$ is closer to $a$ and $b$ than $\operatorname{dist}(a, b)$ Toussaint (1980) (Fig. 9p).

- MST: The Euclidean minimum spanning tree (MST) Nešetřil, Milková, and Nešetřilová (2001) is a connected acyclic graph which has minimum possible sum of edges' lengths (Fig. 9kd). Strictly speaking, the tree rooted in Paris (Fig. 9k) is not the minimum tree however it is just 1.082 longer than the minimum spanning tree rooted in Toulouse (Fig. 9 $\mathrm{d}$ ), see Tab. 1

In general, the graphs relate as $\mathbf{M S T} \subseteq \mathbf{R N G} \subseteq \mathbf{G G}$ Jaromczyk and Toussaint (1992); Matula and Sokal (1980); Toussaint (1980); this is called the Toussaint 


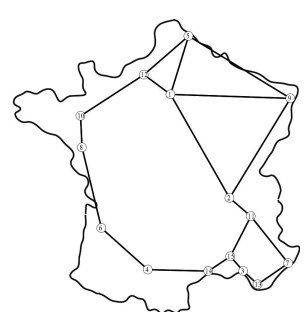

(a) GG

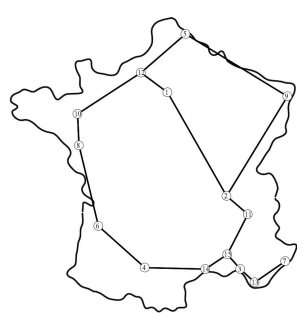

(b) RNG

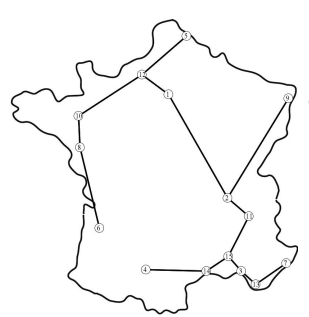

(c) $\mathbf{S T}$ (Paris)

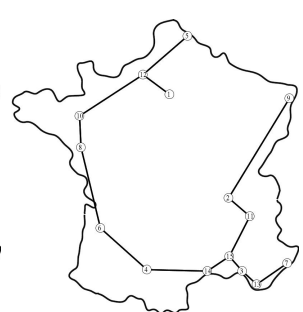

(d) $\operatorname{MST}$ (Toulouse)

Figure 9: Proximity graphs constructed on sites of U. (a) Gabriel graph, (b) relative neighbourhood graph, (c) spanning tree rooted in Paris, (d) minimum spanning tree rooted in Toulouse.

Table 1: Lengths of spanning trees rooted in $\mathbf{U}$.

\begin{tabular}{ll} 
Root & Length \\
\hline 1 & 1.082 \\
2 & 1.138 \\
3 & 1.149 \\
4 & 1.000 \\
5 & 1.084 \\
6 & 1.004 \\
7 & 1.149 \\
8 & 1.110 \\
9 & 1.205 \\
10 & 1.110 \\
11 & 1.138 \\
12 & 1.088 \\
13 & 1.149 \\
14 & 1.149 \\
15 & 1.149
\end{tabular}

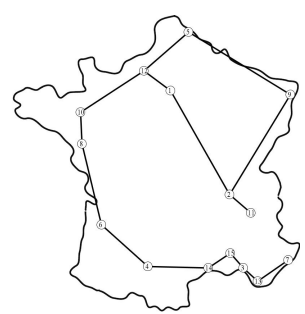

(a) $\mathbf{R N G} \cap \mathbf{H}$

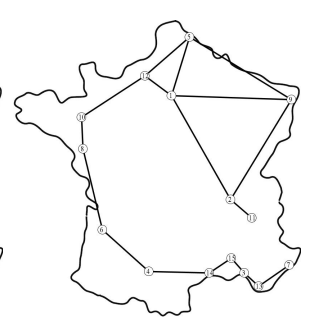

(b) $\mathbf{G G} \cap \mathbf{H}$

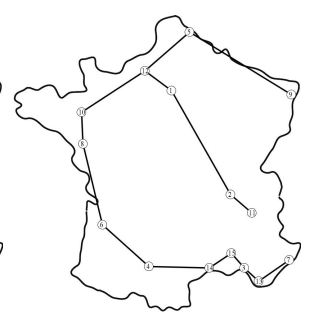

(c) $\mathbf{M S T}$ (Nantes) $\cap \mathbf{H}$

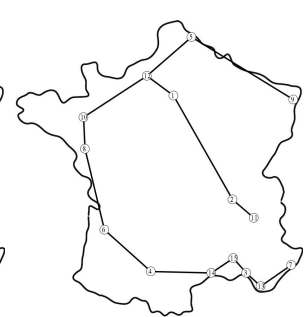

(d) $\mathbf{M S T}$ (Rennes) $\cap \mathbf{H}$

Figure 10: Intersection of highways graph $\mathbf{H}$ with (a) relative neighbourhood graph, (b) Gabriel graph, (c) spanning tree rooted in Nantes, (c) spanning tree rooted in Rennes.

hierarchy.

Intersections of the motorway graph with the proximity graphs is shown in Fig. 10. Neither GG nor RNG are subgraphs of $\mathbf{H}$ however 15 of 17 edges 


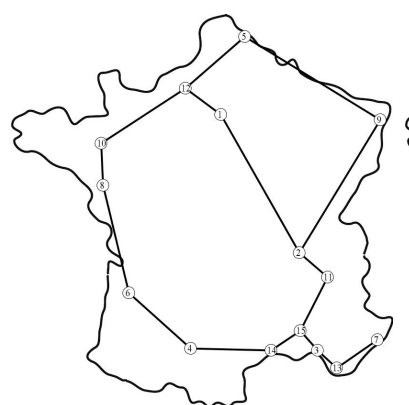

(a) $\mathbf{R N G} \cap \mathbf{P}\left(\frac{1}{12}\right)$

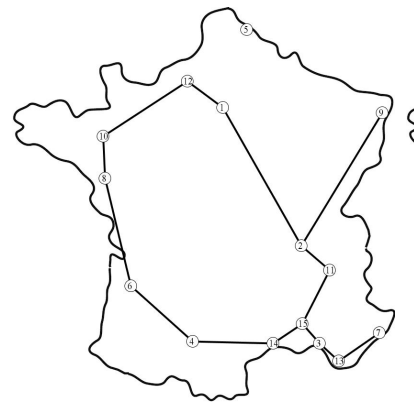

(d) $\mathbf{R N G} \cap \mathbf{P}\left(\frac{6}{12}\right)$

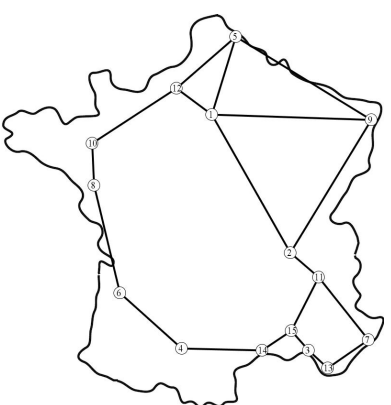

(b) $\mathbf{G G} \cap \mathbf{P}\left(\frac{1}{12}\right)$

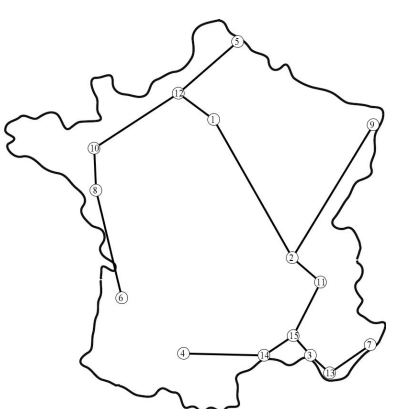

(c) $\mathbf{S T}$ (Paris) $\cap \mathbf{P}\left(\frac{1}{12}\right)$

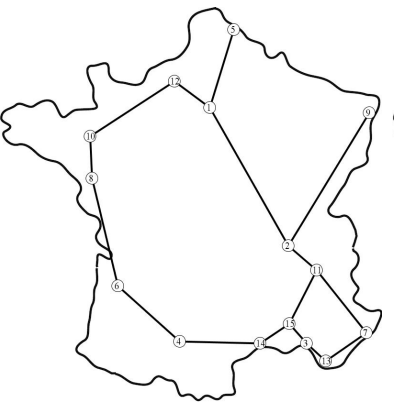

(e) $\mathbf{G G} \cap \mathbf{P}\left(\frac{6}{12}\right)$

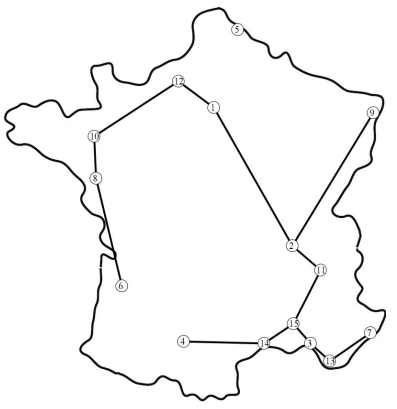

(f) $\mathbf{S T}$ (Paris) $\cap \mathbf{P}\left(\frac{6}{12}\right)$

Figure 11: Intersection of Physarum graphs (abc) $\mathbf{P}\left(\frac{1}{12}\right)$ and (def) $\mathbf{P}\left(\frac{6}{12}\right)$ with (ad) relative neighbourhood graph, (be) Gabriel graph, (cf) spanning tree rooted in Paris,

of $\mathbf{G G}$ are included in $\mathbf{H}$ and 13 of 14 edges of $\mathbf{R N G}$. The missing links are Grenoble-Avignon and Nice-Grenoble in case of GG and Grenoble-Avignon in case of RNG. Only spanning trees rooted in Nantes and Rennes are completely subgraphs of $\mathbf{H}$, intersections of trees rooted in all sites with $\mathbf{H}$ consist of two disconnected components.

We now compare proximity graphs with Physarum graphs $\mathbf{P}$. In Fig. 11 we compare proximity graphs with $\mathbf{P}\left(\frac{1}{12}\right)$ (because this graph represents all links developed in laboratory experiments) and $\mathbf{P}\left(\frac{6}{12}\right)$ (because this graph shows links occurred in over half of the laboratory experiments, and therefore represents the highest accuracy of approximation yet still connected graph).

We find that relative neighbourhood graph RNG, Gabriel graph GG and spanning tree routed in Paris are subgraphs of $\mathbf{P}\left(\frac{1}{12}\right)$ (Fig. 11abc). This indicates that the Physarum graph includes all principal proximity graphs and thus represents an optimal covering of the configuration of major urban areas of France. In contrast, the motorway graph $\mathbf{H}$ does not represent several edges of the proximity graphs, see Fig. 10 .

The high accuracy of representation Physarum graph $\mathbf{P}\left(\frac{6}{12}\right)$ almost includes the proximity (Fig. 11def). Namely, the link Lille-Rouen is mixing from the intersection of the graph $\mathbf{P}\left(\frac{6}{12}\right)$ with RNG, GG and ST. The link StrasbourgRouen is missing from the intersection of the Physarum graph with the relative neighbourhood graph and Gabriel graph. The intersection of $\mathbf{G G}$ and $\mathbf{P}\left(\frac{6}{12}\right)$ is 
also missing the link Paris-Strasbourg.

\section{Computer Modelling of Slime Mould Trans- port Networks}

Since slime mould is a living system it is subject to limitations of repeatability and unpredictability during its foraging, not to mention the long experimental time to form and adapt the transport networks. To aid the analysis of similarities and differences of the slime mould networks with the motorway network we require a computational mechanism to approximate the evolution of Physarum transport networks. Importantly, however, we must ensure that the networks emerge - as with slime mould — from simple, local, and 'bottom-up' interactions, as opposed to the 'top-down' network constructs formed from classical algorithmic approaches.

We use the multi-agent approach that was introduced in Jones (2010) to approximate the emergent transport networks of slime mould. This approach has been demonstrated to be useful for approximating the biological behaviour of slime mould Baumgarten, Jones, and Hauser (2015), Jones (2015b) and in developing biologically inspired unconventional computing methods Jones (2015a). A population of mobile particles is created and initialised on a $2 \mathrm{D}$ lattice configured to the experimental pattern of French cities. The diffusive medium is represented by a discrete two-dimensional floating point lattice. Particle positions are stored on a discrete lattice isomorphic to the diffusive lattice. Particles also store internal floating point representations of position and orientation which are rounded to discrete values to compute movement updates and sensory inputs. A single particle, and an aggregation of particles, are related to the $P$. polycephalum plasmodium in the following way: the plasmodium is conceptualised as an aggregate of identical components. Each particle represents a hypothetical unit of gel/sol interaction. Gel refers to the relatively stiff spongelike matrix composed of actin-myosin fibres and sol refers to the protoplasmic solution which flows within the matrix. The structure of the protoplasmic network is indicated by the particle positions and the flux of sol within the network is represented by the movement of the particles. The resistance of the gel matrix to protoplasmic flux of sol is generated by particle movement collisions. For a more detailed description of the model implementation, see Jones (2015a).

The topology of $P$. polycephalum transport networks is, in part, influenced by unpredictable influences on its formation, for example the initial migration direction of the plasmodium or the presence of previously laid down protoplasmic tubes. This raises the question as to whether the topology of the networks would be more regular under idealised adaptation conditions. In order to minimise this unpredictability, we initialised the simulation model with a uniform distribution of a fully grown virtual plasmodium to solely assess the effect of the spatial arrangement of nutrients (corresponding to city locations) had on the morphological adaptation of the virtual transport network. The results of an example evolution of the virtual plasmodium are shown in Fig. 12. Uniform coverage was attained by populating $50 \%$ of the habitable area of France with 20,000 particles.

The simulation was started and the collective adapted to the nutrient stim- 

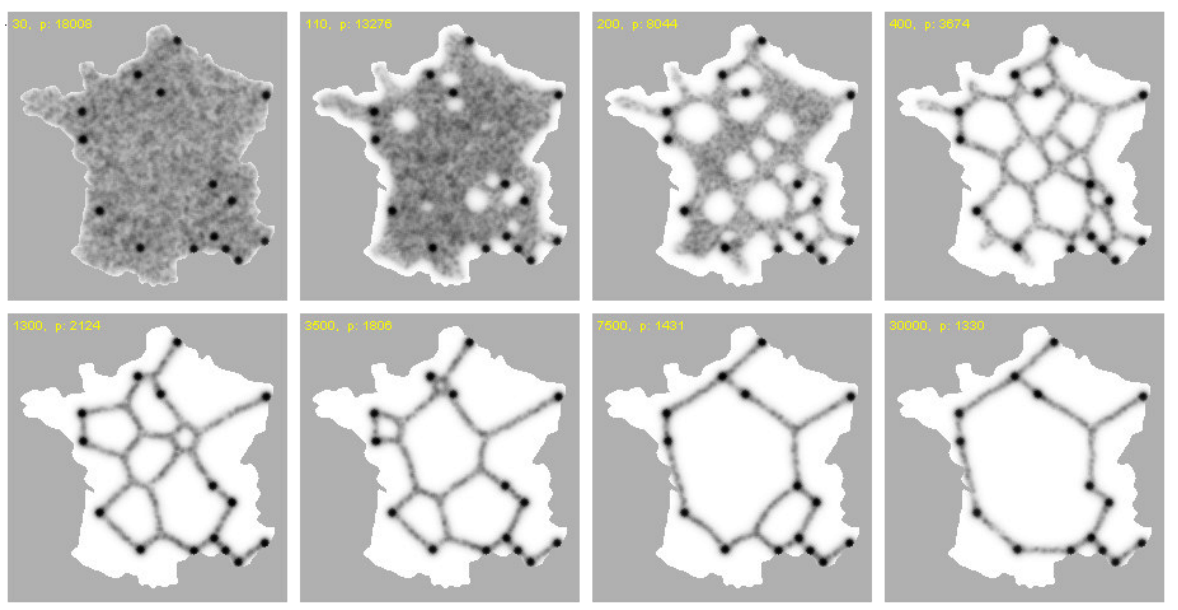

Figure 12: Example experimental run showing adaptation of model plasmodium to the 20 cities, forming a transport network connecting the cities.

ulus by shrinking in size to form a transport network connecting the regions. Twenty runs of the simulation were performed at both fast and slow shrinkage speeds. In the fast shrinkage method, small defects began to form in the material, forming holes which increased in size (Fig. 12). This approximates the self-organised formation of $P$. polycephalum transport networks from a solid initial mass of plasmodium. The model plasmodium maintains its connection to the nutrients (regions) and the transport network adapts its configuration until a stable state is reached. The final networks are typically cyclic (encompassing all regions in a ring structure), with some left-right connectivity between Western and Eastern regions.

In the slower shrinkage method, defects do not form in the virtual plasmodium as it shrinks in size. The final network patterns tend to be as a result of shrinking concave regions in the original shape and approximate Minimum Steiner Trees.

We used the same method of assessing connectivity between cities as in the experimental approach and this resulted in an adjacency matrix containing the frequencies of connected nodes for the 20 experiments in both nutrient conditions. The connectivity graphs at different threshold weights are shown in Fig. 13 for fast shrinkage concentrations and Fig. 14 for slow shrinkage conditions. The fast shrinkage method has a higher average mean degree over all experiments than the slow shrinkage method (degree 2.75 vs 2.12 ). There is a greater connectivity between left and right regions on the map, which persists until the threshold of 14 is reached (above this threshold, the connectivity is similar to the slow method).

A comparison of the connectivity between individual cities is shown in Fig. 15 under fast and slow shrinkage conditions. The most highly connected regions in the fast shrinkage method are Paris, Lyon, Bordeaux and Nantes. It is the connectivity from these regions which is responsible for the West-East crossings. In comparison the most highly connected regions in the slow method are Paris, Lyon, Avignon and Rouen, which are typically connected in a simple path on 

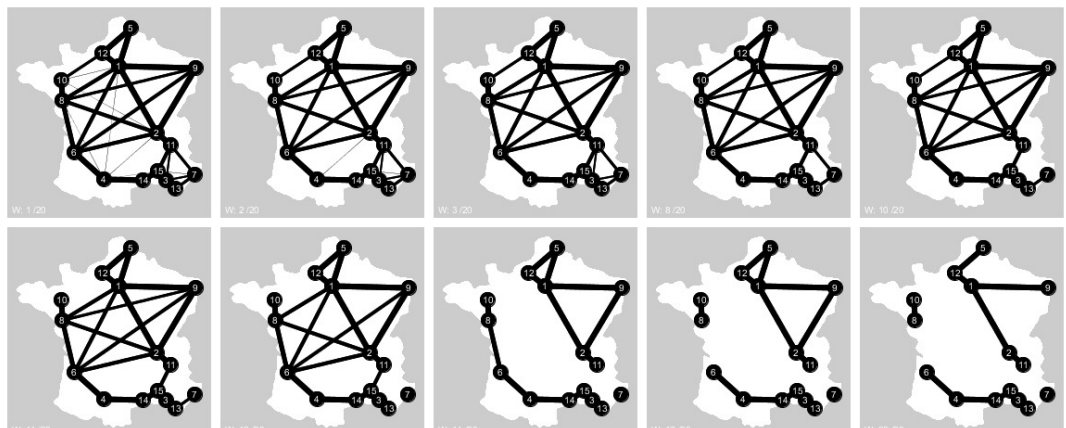

Figure 13: Changes in model plasmodium connectivity as weighting parameter is increased. Images show connectivity at $\mathrm{w}=1,2,3,8,10,11,12,14,17$ and 20. Fast shrinkage conditions.
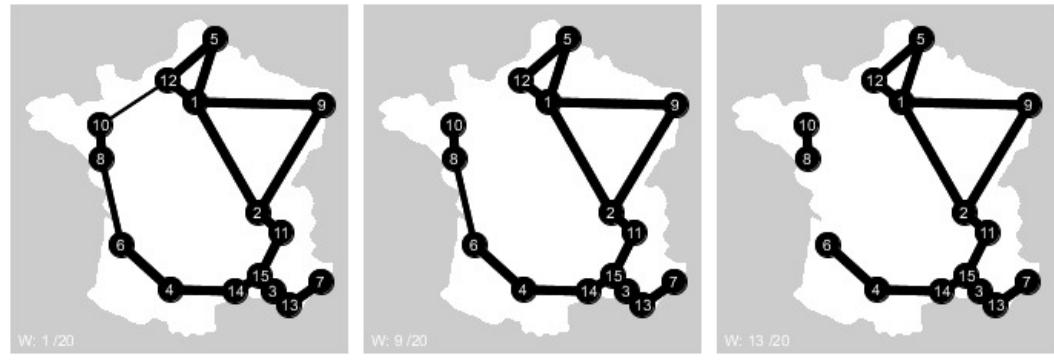

Figure 14: Changes in model plasmodium connectivity as weighting parameter is increased. Images show connectivity at $\mathrm{w}=1,9$ and 20. Slow shrinkage conditions. 


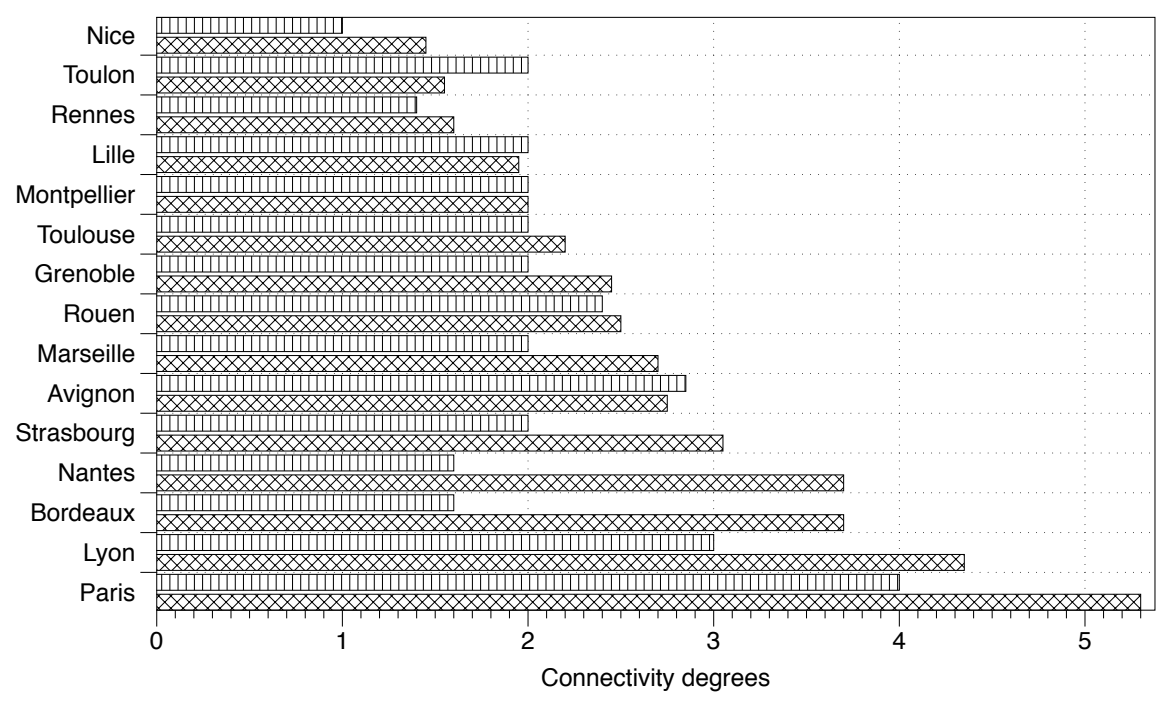

Figure 15: Comparison of connectivity degree of cities during fast and slow shrinkage. Cross-hatched bars indicate fast shrinkage, vertical-hatched bars indicate slower shrinkage.

the Easterly side. The connectivity of Bordeaux and Nantes regions in the fast method (both having mean degree of 3.7) is more than halved (mean degree 1.6) in the slow method, due to the lack of West-East crossings.

\section{Discussions}

Using slime mould $P$. polycephalum we produced an unconventional computing model that imitated development of the French transport network through the distribution of its protoplasmic tubes. We found that at least once during laboratory experiments, slime mould represents over $70 \%$ of man-made motorways in France. Over half of the motorway links are represented by the slime mould in over half of the laboratory experiments. The transport links represented in over $70 \%$ of experiments, i.e. the strongest links, are the chain Lyon-Grenoble and the chain Lille-Paris-Rouen-Rennes-Nantes-Bordeaux-Toulouse-MontpellierAvignon-Marseille-Toulon-Nice. This is a chain of motorways spanning France from the North to South-West to South and South-East.

In comparison to proximity graphs we found that The motorway graphs provide a weak representation of proximity graphs: only spanning trees rooted in Nantes and Rennes are sub-graphs of the motorway graph. Some edges in the relative neighbourhood graph and the Gabriel graph are not included in the motorway graph. In contrast, at least its weakest version, the Physarum graph includes all three types of proximity graphs. Moreover, in the strongest Physarum graph, only a few edges of the proximity graphs are not included. These findings might demonstrate that while French motorways are lacking the proximity logic typical of engineering projects, their topology is supported by 
bio-logic of the slime mould.

We must also note that these results can only be an approximation, since only the 15 most populated urban areas have been taken into account for the experiments to simplify the procedure. Notably, none of the hubs of activity are located in the central area of France. For instance in the Physarum models, Clermont-Ferrand and Limoges are connected by motorways to Paris but rank respectively $19^{\text {th }}$ and $37^{\text {th }}$ in term of population - and Le Havre, ranks $34^{\text {th }}$ but is the $2^{\text {nd }}$ most important harbour in France and therefore has already been connected to Paris by a motorway in the 70s. Perhaps an alternative approach would be to establish a different city ranking for modelling. For example, a more comprehensive selection criteria that takes into account key data such as - population, geographic, historic, tourist and economic criteria - may allow a better representation of the French network without requiring every conurbation to be modelled.

Moreover, limitations were encountered since the experiments were conducted on flat agar plates, where no landscape features (e.g. mountains or lakes) were presented. In future studies it may be possible to incorporate geographic challenges by cultivating the slime mould on three-dimensional templates of France.

Modelling the experimental setup using similar 'bottom-up' local interactions within the virtual plasmodium yielded networks which either retained the central East-West crossing points (fast network adaptation condition) or did not contain the East-West crossing points (slow network adaptation condition). The difference between the two conditions can be interpreted due to the tradeoff between network connectivity and network length. Fast shrinkage conditions resulted in defects in the material, causing increased connectivity (and degree) at the 'expense' of greater network length (i.e. more network material needed). Slower adaptation resulted in fewer defects and shorter overall networks approximating spanning trees, but at the expense of decreased connectivity. A similar trade-off occurs in human constructed motorway networks. It is technically theoretically feasible to construct motorways where each urban area is connected to all other areas. Of course this would practical problems in terms of expense and construction disruption and would contain many redundant or unnecessary links. At the other extreme it would be possible to construct a minimal distance, spanning tree-like network structure. Such a topology, however, would not account for the relative importance between certain links on the tree and would possess too little network reduncancy to cope with network disruption or disconnection. Both Physarum networks and the motorway network attempt to resolve the trade-off between network connectivity and network length.

A fundamental assumption underpinning these experiments is that manmade motorway networks still have many things to learn from networks encountered in Nature such as, the striking spider orb-web appearance of the French motorways. This impression is conferred by the radial distribution of central routes that originate from the capital while peripheral motorways appear to be organized around this powerful hub as concentric circles. Moreover, the density of concentric motorways increases as one approaches Paris (Fig. 1). 


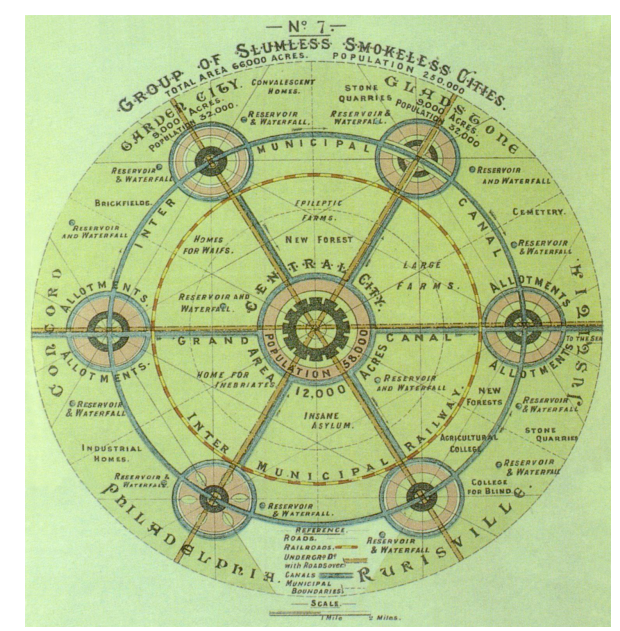

Figure 16: Idealised city-planning designs such as Ebenezer Howard's Garden City Howard (1965) also followed spider-web pattern (image from: http://upload.wikimedia.org/wikipedia/commons/3/3d/Garden _City_Concept_by_Howard.jpg).

However, such analogies may equally well be conferred by other factors such as, the geometry of the environment. This is the most likely explanation for the derived network topologies since the overall geographic region of France is already shaped in an approximately hexagonal configuration - being bounded at the coastal zones and the mountain ranges - and is likely to contribute significantly to the solution produced by the plasmodium network morphology.

For a further study, it would be interesting to experiment whether a spider would construct a similar network structure given the same boundary constraints as topographically delineated at the French frontiers. And secondly, it would be interesting to investigate whether slime mould protoplasmic network can converge freely to the same optimized solution given a proper list of French cities and three-dimensional templates of France. This would allow a better understanding of the optimization process taking place and to improve computer modelling and answer the question: can the spider web and slime mould structures and functions become a fruitful suggestion box for human-made networks?

From an applied perspective Physarum appears to have a deductive, rather than a predictive value in establishing the efficiencies in urban transport routes. Its value in the planning of national highways and motorways therefore is currently limited. Yet, such unconventional computing models may play a role in establishing network optimizations. For example, in developing bypasses - especially if it was demonstrated that replication of 3D topographies can effectively solve the kinds of geographical challenges encountered in this study i.e. the presence of a mountain range.

Physarum demonstrates a striking capacity to deduce wheel and spoke architypes that are generated by highly stable circular and radial forms. These 
are described earlier in this paper as characteristic 'spider web' designs but such idealised forms are also recognised in city planning in projects such as, Ebenezer Howard's Garden City Howard (1965) (see, Fig. 16) and are fully operational in places such as, Vienna where wheel and spoke forms of urban organization and transport organisation have been associated with efficient distribution infrastructures Cook and Goodwin (2008). However, drawbacks of this form of organization include its centralized character that renders day-to-day operations as being relatively inflexible and often requiring two journeys through intersections to reach most destinations. Such an organization system is prone to bottlenecks and operational congestion.Potentially this may explain why, when an obstacle such as, a mountain range is reached, that no robust alternative transport route is proposed.

Perhaps the ultimate test of a Physarum transport network may be to ask it to deduce a grid structure which is typical of New York or Milton Keynes in the UK. These road structures were designed for maximum navigation and efficiency so, the comparisons between human logic and natural logic in developing transport solutions may be most optimally compared in these systems - particularly as they are driven by geometrical propositions and have comparatively little historical complexity, or 'organic logic' in their evolution. Potentially by working at different scales and introducing $3 \mathrm{D}$ challenges into the nutrient matrix using $3 \mathrm{D}$ printing techniques it may be possible to identify more strategic applications of Physarum in the design of city transport systems that may favour stable network typologies.

\section{References}

Adamatzky, A. (2010). Physarum machines: Computers from slime mould (Vol. 74). World Scientific Pub Co Inc.

Adamatzky, A. (2012). Bioevaluation of world transport networks. World Scientific.

Adamatzky, A., Akl, S., Alonso-Sanz, R., Van Dessel, W., Ibrahim, Z., Ilachinski, A., ... others (2013). Are motorways rational from slime mould's point of view? International Journal of Parallel, Emergent and Distributed Systems, 28(3), 230-248.

Adamatzky, A., Bull, L., \& Costello, B. D. L. (2007). Unconventional computing 200\%. Luniver Press.

Adamatzky, A., \& Jones, J. (2010). Road planning with slime mould: If Physarum built motorways it would route M6/M74 through newcastle. International Journal of Bifurcation and Chaos, 20(10), 3065-3084.

Adamatzky, A. I. (2014). Route 20, autobahn 7, and slime mold: Approximating the longest roads in usa and germany with slime mold on 3-d terrains. Cybernetics, IEEE Transactions on, 44(1), 126-136.

ASFA. (2014). Chiffres clés 2014, asfa (association professionnelle des sociétés françaises concessionnaires ou exploitantes d'autoroutes ou d'ouvrages routiers) [Computer software manual]. Retrieved from http://www . autoroutes.fr/

Baumgarten, W., Jones, J., \& Hauser, M. (2015). Network coarsening dynamics in a plasmodial slime mould: Modelling and experiments. Acta Physica 
Polonica B, 46(6), In-press.

Bebber, D., Hynes, J., Darrah, P., Boddy, L., \& Fricker, M. (2007). Biological solutions to transport network design. Proceedings of the Royal Society B: Biological Sciences, 274(1623), 2307-2315.

Cook, G. N., \& Goodwin, J. (2008). Airline networks: A comparison of hub-andspoke and point-to-point systemsairline networks: A comparison of huband-spoke and point-to-point systems. The Journal of Aviation/Aerospace Education 83 Research, 17(2), 1.

Durham, A., \& Ridgway, E. (1976). Control of chemotaxis in Physarum polycephalum. The Journal of Cell Biology, 69, 218-223.

Fricker, M. D., Bebber, D., \& Boddy, L. (2008). Mycelial networks: structure and dynamics. In British mycological society symposia series (Vol. 28, pp. $3-18)$.

Gabriel, K., \& Sokal, R. (1969). A new statistical approach to geographic variation analysis. Systematic Biology, 18(3), 259.

Gandil, P. (2005). Development of the motorway infrastructure in france: background and recent developments. In The 13th annual conference on public-private partnerships, toronto, ontari (p. 3).

Garnier, S., Guérécheau, A., Combe, M., Fourcassié, V., \& Theraulaz, G. (2009). Path selection and foraging efficiency in argentine ant transport networks. Behavioral Ecology and Sociobiology, 63(8), 1167-1179.

Howard, E. (1965). Garden cities of to-morrow (Vol. 23). Mit Press.

Jaromczyk, J., \& Toussaint, G. (1992). Relative neighborhood graphs and their relatives. Proceedings of the IEEE, 80(9), 1502-1517.

Jones, J. (2010). The emergence and dynamical evolution of complex transport networks from simple low-level behaviours. Int. Journal of Unconventional Comput., 6, 125-144.

Jones, J. (2015a). From pattern formation to material computation: Multi-agent modelling of physarum polycephalum (Vol. 15). Springer.

Jones, J. (2015b). Mechanisms inducing parallel computation in a model of physarum polycephalum transport networks. Parallel Processing Letters, $25(01), 1540004$.

Matula, D., \& Sokal, R. (1980). Properties of gabriel graphs relevant to geographic variation research and the clustering of points in the plane. Geographical Analysis, 12(3), 205-222.

Nakagaki, T., Yamada, H., \& Toth, A. (2000). Intelligence: Maze-solving by an amoeboid organism. Nature, 407, 470.

Nešetřil, J., Milková, E., \& Nešetřilová, H. (2001). Otakar borvka on minimum spanning tree problem translation of both the 1926 papers, comments, history. Discrete Mathematics, 233(1), 3-36.

Reid, C., Latty, T., Dussutour, A., \& Beekman, M. (2012). Slime mold uses an externalized spatial memory to navigate in complex environments. Proceedings of the National Academy of Sciences, 109(43), 17490-17494.

Saigusa, T., Tero, A., Nakagaki, T., \& Kuramoto, Y. (2008). Amoebae anticipate periodic events. Phys. Rev. Lett., 100(1). doi: 10.1103/ PhysRevLett.100.018101

Shirakawa, T., Adamatzky, A., Gunji, Y.-P., \& Miyake, Y. (2009). On simultaneous construction of voronoi diagram and delaunay triangulation by Physarum polycephalum. International Journal of Bifurcation and Chaos, 19(9), 3109-3117. 
Tero, A., Takagi, S., Saigusa, T., Ito, K., Bebber, D. P., Fricker, M., ... Nakagaki, T. (2010). Rules for biologically inspired adaptive network design. Science, 327(5964), 439-442.

Toussaint, G. (1980). The relative neighbourhood graph of a finite planar set. Pattern Recognition, 12(4), 261-268. 